\title{
Physical rehabilitation for critical illness myopathy and neuropathy (Protocol)
}

\author{
Mehrholz J, Pohl M, Kugler J, Burridge J, Mückel S
}

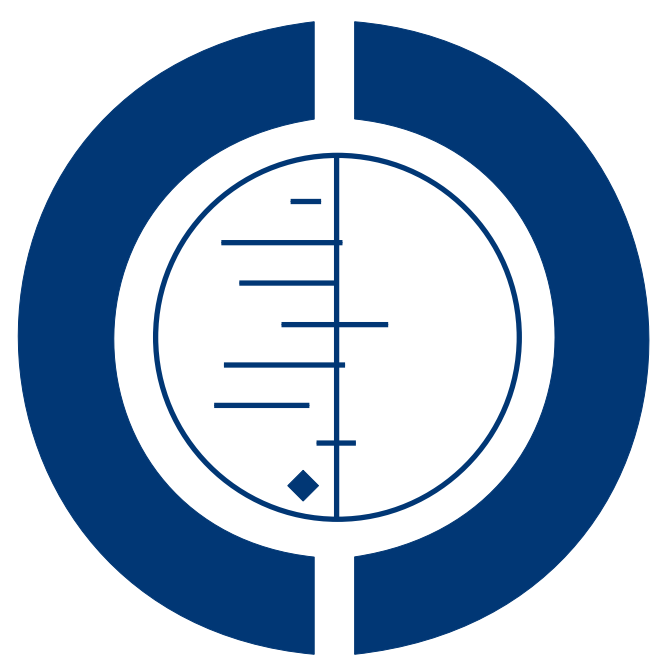

\section{THE COCHRANE COLLABORATION $^{\circledR}$}

This is a reprint of a Cochrane protocol, prepared and maintained by The Cochrane Collaboration and published in The Cochrane Library 2014, Issue 1

http://www.thecochranelibrary.com

\section{WILEY}


TABLE OF CONTENTS

HEADER . . . . . . . . . . . . . . . . . . . . . . . . . . . . . . . . . . . . 1

ABSTRACT . . . . . . . . . . . . . . . . . . . . . . . . . . . . . . . . . . . . . . 1

BACKGROUND . . . . . . . . . . . . . . . . . . . . . . . . . . . . . . . . . . . .

OBJECTIVES . . . . . . . . . . . . . . . . . . . . . . . . . . . . . . . . . . . . . . .

METHODS . . . . . . . . . . . . . . . . . . . . . . . . . . . . . . . . . . . . . .

ACKNOWLEDGEMENTS . . . . . . . . . . . . . . . . . . . . . . . . . . . . . . . . . . . . . . .

REFERENCES . . . . . . . . . . . . . . . . . . . . . . . . . . . . . . . . . . . . . . 6

APPENDICES . . . . . . . . . . . . . . . . . . . . . . . . . . . . . . . . . . . . . . 7

CONTRIBUTIONS OF AUTHORS . . . . . . . . . . . . . . . . . . . . . . . . . . . . . . . . . . . . . . . . . .

DECLARATIONS OF INTEREST . . . . . . . . . . . . . . . . . . . . . . . . . . . . . . . . . . 8

SOURCES OF SUPPORT . . . . . . . . . . . . . . . . . . . . . . . . . . . . . . . . . . . . . . . . . . . . . .

Physical rehabilitation for critical illness myopathy and neuropathy (Protocol)

Copyright $\odot 2014$ The Cochrane Collaboration. Published by John Wiley \& Sons, Ltd. 


\title{
[Intervention Protocol] \\ Physical rehabilitation for critical illness myopathy and neuropathy
}

Jan Mehrholz ${ }^{1,2}$, Marcus Pohl ${ }^{3}$, Joachim Kugler ${ }^{2}$, Jane Burridge $^{4}$, Simone Mückel ${ }^{1}$

${ }^{1}$ Wissenschaftliches Institut, Private Europäische Medizinische Akademie der Klinik Bavaria in Kreischa GmbH, Kreischa, Germany. ${ }^{2}$ Department of Public Health, Dresden Medical School, Technical University Dresden, Dresden, Germany. ${ }^{3}$ Abteilung Neurologie und Fachübergreifende Rehabilitation, Klinik Bavaria Kreischa, Kreischa, Germany. ${ }^{4}$ Research Group, Faculty of Health Sciences, University of Southampton, Southampton, UK

Contact address: Jan Mehrholz, jan.mehrholz@klinik-bavaria.de.

Editorial group: Cochrane Neuromuscular Disease Group.

Publication status and date: New, published in Issue 1, 2014.

Citation: Mehrholz J, Pohl M, Kugler J, Burridge J, Mückel S. Physical rehabilitation for critical illness myopathy and neuropathy. Cochrane Database of Systematic Reviews 2014, Issue 1. Art. No.: CD010942. DOI: 10.1002/14651858.CD010942.

Copyright (C) 2014 The Cochrane Collaboration. Published by John Wiley \& Sons, Ltd.

\begin{abstract}
A B S T R A C T
This is the protocol for a review and there is no abstract. The objectives are as follows:

To assess the effects of rehabilitation therapies for people with CIP and CIM.

The primary objective is to assess the effectiveness of such interventions in improving daily activities such as walking, bathing, dressing and eating.
\end{abstract}

Secondary objectives are to assess effects on muscle strength and quality of life, and to assess adverse effects of rehabilitation.

\section{B A C K G R O U N D}

\section{Description of the condition}

Critical illness myopathy (CIM) and polyneuropathy (CIP) are common complications of critical illness that frequently occur together. Both conditions cause weakness of limb and respiratory muscles, which increase morbidity and impede recovery. CIP is a distal axonal sensory-motor polyneuropathy, which means that multiple nerves that control movement and carry sensation are affected. CIP presents as limb and respiratory muscle weakness (Fan 2012; Lacomis 2000), and is closely associated with a failure to wean patients from mechanical ventilation (Fan 2012; Latronico 2011). The diagnostic criteria for CIP as a dysfunction of (multiple) peripheral nerves are, according to Bolton 2005 and Latronico 2011, as follows.

1. The individual is critically ill (multi-organ dysfunction and failures).

2. The individual has limb weakness, or is difficult to wean from a ventilator (after exclusion of non-neuromuscular causes such as heart and lung disease).

3. There is electrophysiological evidence of axonal motor and sensory polyneuropathy.

4. There is an absence of a decremental response on repetitive nerve stimulation (Latronico 2011).

A definite diagnosis of CIP, according to Bolton 2005 and Latronico 2011, is established if all four criteria are fulfilled. A probable diagnosis of CIP is established if criteria one, three and 
four are fulfilled.

CIM is a primary myopathy (that is, a muscle disorder) that is not secondary to muscle denervation, and which has characteristic electrophysiological and morphological changes (Latronico 2011). The diagnostic criteria for critical illness myopathy are, according to Latronico 2011, as follows.

1. The individual is critically ill (multi-organ dysfunction and failures)

2. The individual has limb weakness, or is difficult to wean from a ventilator (after exclusion of non-neuromuscular causes such as heart and lung disease).

3. Compound muscle action potential (CMAP) amplitudes less than $80 \%$ of the lower limit of normal are recorded in two or more nerves without conduction block.

4. Sensory nerve action potential amplitudes are more than $80 \%$ of the lower limit of normal.

5. Needle electromyography has detected:

i) short duration, low-amplitude motor unit potentials with early or normal full recruitment, with or without fibrillation potentials in conscious and cooperative patients; or

ii) increased CMAP duration or reduced muscle

membrane excitability on direct muscle stimulation in noncooperative patients.

6. An absence of a decreasing response on repetitive nerve stimulation.

7. Muscle histopathological findings of primary myopathy (for example, myosin loss or muscle necrosis).

Fulfillment of all criteria establishes a definite diagnosis of CIM (Latronico 2011). Fulfillment of criteria one and three to six establishes a probable diagnosis of CIM. For our review we will apply these definitions of CIP and CIM.

The pathophysiology of CIP and CIM is complex and remains unclear (Hermans 2008). The development of CIP and CIM is thought to involve electrical, microvascular, metabolic and bioenergetic pathophysiological mechanisms (see Hermans 2008 for a good overview). Crucial risk factors for CIP and CIM are sepsis, systemic inflammatory response syndrome, and multiple organ failure (Hermans 2008).

Exact numbers and rates of CIP and CIM are not well established because of variations in the patient population, in risk factors and in the diagnostic and assessment criteria used. In people receiving mechanical ventilation or with an increased risk of developing multi-organ failure, the risk of developing CIP or CIM is about $30 \%$ and the risk increases up to $50 \%$ in people with acute respiratory distress syndrome (Hermans 2008). The risk of developing CIP or CIM is associated with a lengthy (more than one week) stay in the intensive care unit (ICU), multi-organ failure (with or without sepsis) and systemic inflammatory response syndrome (Bercker 2005; Hermans 2008). A systematic review estimated the incidence of CIP and CIM at 46\% (95\% CI 43\% to $49 \%$ ) among adults who had remained in the ICU for more than two weeks, who were receiving mechanical ventilation and had sepsis or multi-organ failure (Stevens 2007).

The short- and long-term impact of CIP and CIM are well described (Herridge 2011; Ohtake 2012; Hermans 2008). The muscle weakness associated with these conditions can prolong the need for supported ventilation and delay weaning from a ventilator. In turn, this can mean a longer stay in the ICU and in hospital, and slower rehabilitation (Hermans 2008; Zanni 2010). Although full recovery has been reported in approximately $50 \%$ of people with CIM/CIP, improvement is related to the severity of the condition. People who are only mildly affected can recover within weeks, but those with more severe weakness may take months to improve, or in some cases, remain severely affected with partial or no recovery (Hermans 2008). Clinical and neurophysiological signs may remain for up to five years after discharge from hospital and may lead to long-term paresis or paralysis (Herridge 2011).

Generalised weakness syndromes such as CIP and CIM are a major cause of chronically impaired motor function that can affect activities of daily living and participation. Physical rehabilitation of those affected is therefore of great importance and interest.

\section{Description of the intervention}

In the last five years, research has suggested that physical rehabilitation may improve activities in people with CIP and CIM (NortonCraft 2012; Connolly 2012; Ohtake 2012; Bemis-Dougherty 2012; Fan 2012). Rehabilitation interventions include physical and occupational therapy techniques, such as stretching, strength training and mobility training (for example, training in dressing and transfers, or to improve balance, sit-to-stand, and walking) (Burtin 2009; Hanekom 2011; Herridge 2011; Fan 2012; Norton-Craft 2012). There has, however, been no Cochrane review of the available literature on the efficacy and acceptability of physical rehabilitation approaches to treat people who have developed CIP and CIM.

\section{Why it is important to do this review}

Advances in medical care, increased survival rates and better diagnosis of CIP and CIM, as well as the availability of more effective therapy, have highlighted the need for and potential value of rehabilitation for people with critical illness. However, although rehabilitation guidelines give recommendations, there is limited evidence on which to base practice (Connolly 2012). People with ICU-acquired weakness may be most likely to benefit from ongoing rehabilitation (Connolly 2012). A recent special issue in the journal Physical Therapy underlined the importance of the topic; the authors concluded that physical rehabilitation approaches for improving activities after CIP/CIM showed some promising signs of effectiveness (Bemis-Dougherty 2012; Norton-Craft 2012), but there was a need for more evidence to provide clear guidelines. 
In addition to the question of whether physical rehabilitation in general is beneficial, it is still unclear which specific rehabilitation approaches are effective, and the most appropriate frequency and intensity with which to apply them in practice (Connolly 2012). There is currently no Cochrane systematic review in this area. We therefore aim, in this Cochrane review, to compile and assess all the available literature about the effectiveness and safety of physical rehabilitation in CIP and CIM, so as to provide comprehensive, up-to-date evidence on which to base clinical practice.

\section{O B J E C T IVES}

To assess the effects of rehabilitation therapies for people with CIP and CIM.

The primary objective is to assess the effectiveness of such interventions in improving daily activities such as walking, bathing, dressing and eating.

Secondary objectives are to assess effects on muscle strength and quality of life, and to assess adverse effects of rehabilitation.

\section{METHODS}

\section{Criteria for considering studies for this review}

\section{Types of studies}

We will consider randomised controlled trials (RCTs), quasi-RCTs and randomised controlled cross-over trials. Quasi-RCTs are trials in which the method used to allocate participants to interventions is not truly random (for example, using date of birth or hospital number).

\section{Types of participants}

We will include participants aged 18 and over in both inpatient and outpatient settings, who have generalised weakness syndrome and a confirmed or probable diagnosis of CIP or CIM. We will include studies with mixed populations of people with neurological conditions if we are able to obtain the data for participants with generalised weakness syndrome due to CIP or CIM.

\section{Types of interventions}

We will include all trials of CIM and CIP that compare any rehabilitation intervention, such as physiotherapy or occupational therapy, or both, with any other interventions for improving function and activity. Possible and typical interventions in this review might be cycling, sit-to-stand training, walking and gait training, and neuromuscular electrical stimulation to lower extremity muscle groups.

We will include all studies of inpatient and outpatient physical rehabilitation in the acute and chronic phase that compare two or more interventions; for example, neuromuscular electrical stimulation versus usual therapy, or neuromuscular electrical stimulation versus another active intervention (such as muscle strength training, dressing exercises, or gait training). The intervention and control group must have received the same (standard) medication or rehabilitation. We will therefore allow co-interventions as long as they are provided in the same amount to all randomised participants.

\section{Types of outcome measures}

The time frame for outcome measurement will be immediately after the intervention and at follow-up one year later.

\section{Primary outcomes}

Our primary outcome measure will be activities (for example, mobility, walking, transfers, and self care), as measured by validated outcome tools.

Measurement tools will be the Functional Independence Measure (FIM) (Dodds 1993), the Barthel Index (Mahoney 1965) and other validated scales for the ICU such as the Functional Status Score for ICU (FSS-ICU) (Zanni 2010), the Acute Care Index of Function (ACIF) (Roach 1988), the Physical Function Outcome Measure (PFIT) (Skinner 2009), or other validated measures. Measures of walking ability will be gait speed in $\mathrm{m} / \mathrm{s}$ and six-minute walking distance (Norton-Craft 2012).

If studies use multiple scales to measure our primary outcome, we will, in the absence of our preferred measures, list and report all scales and measures used in these studies.

\section{Secondary outcomes}

We will report the following secondary outcomes.

1. Muscle strength (as measured by, for example, the Medical Research Council (MRC) Scale for Muscle Strength (Fan 2012)).

2. Quality of life measured by validated measures, such as the Short Form-36 Health Survey (SF-36) (Skinner 2011), the RAND-36 (Kaarlola 2006), or the Assessment of Quality of Life (AQol) Utility Instrument (Denehy 2008).

3. Frequency of adverse effects as a result of the intervention. We will also report all serious adverse events, defined as those events that are life-threatening, require prolonged hospitalisation, or are fatal. We will report adverse events that occur during the intervention or at less than 12 months after study onset. We will report any information on costs in the 'Discussion'. 


\section{Outcomes for inclusion in a 'Summary of findings table'}

We will use GRADEpro (GRADEpro 2008) software and create a 'Summary of findings' table with the following outcomes: activities, muscle strength, quality of life and adverse effects. Time points for all outcomes in the 'Summary of findings' table will be immediately after the intervention and at follow-up one year later. We will use the five GRADE considerations (study limitations, consistency of effect, imprecision, indirectness and publication bias) to assess the quality of a body of evidence (studies that contribute data for the prespecified outcomes). We will use methods and recommendations described in Chapter 12 of the Cochrane Handbook for Systematic Reviews of Interventions (Higgins 2011). We will justify all decisions to down- or up-grade the quality of evidence using footnotes.

\section{Search methods for identification of studies}

\section{Electronic searches}

We will search the Cochrane Neuromuscular Disease Group Specialized Register, CENTRAL (The Cochrane Library current issue), MEDLINE (1966 to present), EMBASE (1980 to present), CINAHL (from 1982), and the Physiotherapy Evidence Database (PEDro, http://www.pedro.org.au/). We will use the search strategy in Appendix 1 to search MEDLINE and will modify it for the other databases. There will be no language restrictions.

We will search ClinicalTrials.gov (http://clinicaltrials.gov/), the World Health Organization International Clinical Trials Registry Platform (ICTRP) portal (http://www.who.int/ ictrp/en/) and the European Union Clinical Trials Register ( www.clinicaltrialsregister.eu) for ongoing trials and further data about included studies.

\section{Searching other resources}

We will review the bibliographies of any identified RCTs and contact the authors and known experts in the field to identify additional published or unpublished data.

\section{Data collection and analysis}

\section{Selection of studies}

Two review authors (JM and MP) will independently select the eligible trials based on the predefined selection criteria and will resolve disagreement through discussion with the other review authors. If we need further information, we will contact trial authors.

\section{Data extraction and management}

Two review authors (MP and JM) will independently extract trial and outcome data from the selected trials using a data collection form. If one of the review authors was involved in an included trial, another review author will extract trial and outcome data from this trial.

The review authors will extract the following data (according to the Cochrane Handbook for Systematic Reviews of Interventions Table 7.3.a):

1. source;

2. eligibility;

3. participants (important imbalances in prognostic factors at baseline, country, number of participants, age, gender, inclusion and exclusion criteria, educational background, socioeconomic status, cognition, pre-existing neurological impairment(s), neurological history);

4. interventions;

5. outcomes and their time point of measurement;

6. comparison (details of interventions in treatment and control groups, duration of treatment, details of co-intervention in the groups;

7. results;

8. funding source and conflicts of interest among investigators; and

9. miscellaneous.

Two review authors (MP and JM) will check the extracted data for agreement. If they are not able to reach a consensus, a third review author will arbitrate.

If necessary, the review authors will contact trial authors for more information. We will report the outcome of efforts to source missing data in the review.

One review author (JM) will enter data into the Cochrane authoring and statistical software Review Manager 5 (RevMan) (RevMan 2012), and a second author (MP) will check the data entry.

\section{Assessment of risk of bias in included studies}

Two review authors (JK, MP) will independently assess the risk of bias in the included trials according to the Cochrane Handbook for Systematic Reviews of Interventions (Higgins 2011a).

We will use checklists to independently assess:

- methods of random sequence generation;

- methods of allocation concealment;

- blinding of participants and personnel;

- blinding of outcome assessors;

- incomplete outcome data (dropouts, use of an intention-totreat analysis (ITT));

- selective outcome reporting; and

- other sources of bias.

Our judgements will be "High risk of bias", "Low risk of bias" or "Unclear risk of bias". We will use "Unclear" where there is too little information, or when what happened in the study is known 
but the risk of bias is unclear. We will describe the agreement between authors during the 'Risk of bias' assessment, and we will resolve disagreement by reaching consensus through discussion. We will contact trialists for clarification and to request missing information when necessary.

\section{Measures of treatment effect}

For all outcomes representing continuous data, we will enter means and standard deviations. We will calculate a pooled estimate of the mean difference (MD) with $95 \%$ confidence intervals (CI). If studies assess the same outcome but measure it with different scales, we will calculate standardised mean differences (SMD) instead of MD (Higgins 2011b). For all binary outcomes, we will calculate risk ratios (RR) with $95 \%$ CI. Where trials (or groups within a trial) have no event, e.g. no adverse events, we will calculate risk differences (RD) with 95\% CI instead of RR. For all statistical comparisons we will use RevMan.

\section{Unit of analysis issues}

According to guidance in the Cochrane Handbook for Systematic Reviews of Interventions (Higgins 2011c), we will use only the first period of cross-over trials and analyse the data as a parallel group trial.

\section{Dealing with missing data}

We will contact the relevant principal investigators in order to retrieve missing data. We will report the results of these efforts in the review.

\section{Assessment of heterogeneity}

We will use the Higgins and Thompson's $\mathrm{I}^{2}$ statistic in order to assess heterogeneity (Higgins 2002). The $\mathrm{I}^{2}$ statistic ranges from $0 \%$ to $100 \%$, where $0 \%$ indicates that the size of effects, for example MDs of different studies pooled, are consistent with each other.

If we detect heterogeneity, we will attempt to explore the reasons for it (for example, whether related to characteristics of the studies, such as participants, risk of bias, year of study, or place of study).

\section{Assessment of reporting biases}

We will assess publication bias visually using RevMan funnel plots if the review includes 10 or more studies. However, publication bias is not the only cause of asymmetrical funnel plots.

\section{Data synthesis}

We will use a random-effects model, regardless of the level of heterogeneity found with the $\mathrm{I}^{2}$ statistic. Thus, in cases of heterogeneity, we will not violate the preconditions of a fixed-effect model approach.

If the review includes more than one comparison, which cannot be included in the same analysis, we will report the results for each comparison separately.

\section{Subgroup analysis and investigation of heterogeneity}

We will conduct three subgroup analyses as follows.

1. Duration of illness or critical condition: acute/subacute phase (first six months of initiated intervention) versus chronic phase (illness of more than six months' duration and interventions initiated accordingly).

2. Use of definitions of CIP and CIM: use of definitive definition of CIP and CIM in studies versus use of probable definition.

3. Imbalance of prognostic factors at baseline (such as age, duration of illness and duration of ventilation).

We will consider meta-regression as an extension to subgroup analyses when there are more than 10 studies in a meta-analysis.

\section{Sensitivity analysis}

To test the robustness of results we will conduct a sensitivity analysis, removing studies at high or unclear risk of bias (for example, high or unclear versus low risk of bias from method of randomisation).

\section{ACKNOWLEDGEMENTS}

We thank Ruth Brassington and the Cochrane Neuromuscular Disease Group for giving us helpful support.

Editorial support from the Cochrane Neuromuscular Disease Group is funded by the MRC Centre for Neuromuscular Diseases. 


\section{R E F E R E N C E S}

\section{Additional references}

\section{Bemis-Dougherty 2012}

Bemis-Dougherty AR, Smith JM. What follows survival of critical illness? Physical therapists' management of patients with post-intensive care syndrome. Physical Therapy 2013; 93(2):179-85.

\section{Bercker 2005}

Bercker S, Weber-Carstens S, Deja M, Grimm C, Kaisers $\mathrm{U}$. Critical illness polyneuropathy and myopathy in patients with acute respiratory distress syndrome. Critical Care Medicine 2005;33(4):711-5.

Bolton 2005

Bolton CF. Neuromuscular manifestations of critical illness. Muscle \& Nerve 2005;32(2):140-63.

\section{Burtin 2009}

Burtin C, Clerckx B, Robbeets C, Ferdinande P, Langer $\mathrm{D}$, Troosters $\mathrm{T}$, et al.Early exercise in critically ill patients enhances short-term functional recovery. Critical Care Medicine 2009;37(9):2499-505.

\section{Connolly 2012}

Connolly B, Denehy L, Brett S, Elliott D, Hart N. Exercise rehabilitation following hospital discharge in survivors of critical illness: an integrative review. Critical Care 2012;16 (3):226 [Epub ahead of print].

\section{Denehy 2008}

Denehy L, Berney S, Skinner E, Edbrooke L, Warrillow S, Hawthorne G, et al.Evaluation of exercise rehabilitation for survivors of intensive care: protocol for single blind randomised controlled trial. Open Critical Care Medicine Journal 2008;1:39-47.

\section{Dodds 1993}

Dodds TA, Martin DP, Stolov WC, Deyo RA. A validation of the functional independence measurement and its performance among rehabilitation inpatients. Archives of Physical Medicine and Rehabilitation 1993;74(5):531-6.

Fan 2012

Fan E. Critical illness neuromyopathy and the role of physical therapy and rehabilitation in critically ill patients. Respiratory Care 2012;57(6):933-44; discussion 944-6.

\section{GRADEpro 2008}

Jan Brozek, Andrew Oxman, Holger Schünemann. GRADEpro. 3.2 for Windows. Jan Brozek, Andrew Oxman, Holger Schünemann, 2008.

\section{Hanekom 2011}

Hanekom S, Gosselink R, Dean E, van Aswegen H, Roos $\mathrm{R}$, Ambrosino N, et al.The development of a clinical management algorithm for early physical activity and mobilization of critically ill patients: synthesis of evidence and expert opinion and its translation into practice. Clinical Rehabilitation 2011;25(9):771-87.

\section{Hermans 2008}

Hermans G, De Jonghe B, Bruyninckx F, Van den Berghe G. Clinical review: critical illness polyneuropathy and myopathy. Critical Care 2008;12(6):238.

\section{Herridge 2011}

Herridge MS, Tansey CM, Matté A, Tomlinson G, DiazGranados N, Cooper A, et al.Functional disability 5 years after acute respiratory distress syndrome. New England Journal of Medicine 2011;364(14):1293-304.

\section{Higgins 2002}

Higgins JP, Thompson SG. Quantifying heterogeneity in a meta-analysis. Statistics in Medicine 2002;21(11):1539-58.

\section{Higgins 2011}

Higgins JPT, Green S (editors). Cochrane Handbook for Systematic Reviews of Interventions Version 5.1.0 [updated March 2011]. The Cochrane Collaboration, 2011. Available from www.cochrane-handbook.org.

\section{Higgins 2011a}

Higgins JPT, Altman DG, Sterne JAC (editors). Chapter 8: Assessing risk of bias in included studies. In: Higgins JPT, Green S (editors). Cochrane Handbook for Systematic Reviews of Interventions Version 5.1.0 [updated March 2011]. The Cochrane Collaboration, 2011. Available from www.cochrane-handbook.org.

\section{Higgins 2011b}

Deeks JJ, Higgins JPT, Altman DG (editors). Chapter 9: Analysing data and undertaking meta-analyses. In: Higgins JPT, Green S (editors). Cochrane Handbook for Systematic Reviews of Interventions Version 5.1.0 [updated March 2011]. The Cochrane Collaboration, 2011. Available from www.cochrane-handbook.org.

\section{Higgins 2011c}

Deeks JJ, Higgins JPT, Altman DG (editors). Chapter 16: Special topics in statistics In: Higgins JPT, Green $S$ (editors). Cochrane Handbook for Systematic Reviews of Interventions Version 5.1.0 [updated March 2011]. The Cochrane Collaboration, 2011. Available from www.cochrane-handbook.org.

\section{Kaarlola 2006}

Kaarlola A, Tallgren M, Pettilä V. Long-term survival, quality of life, and quality-adjusted life-years among critically ill elderly patients. Critical Care Medicine 2006;34 (8):2120-6.

Lacomis 2000

Lacomis D, Zochodne DW, Bird SJ. Critical illness myopathy. Muscle \& Nerve 2000;12:1785-8.

Latronico 2011

Latronico N, Bolton CF. Critical illness polyneuropathy and myopathy: a major cause of muscle weakness and paralysis. Lancet Neurolology 2011;10(10):931-41. 
Mahoney 1965

Mahoney FI, Barthel DW. Functional evaluation: the Barthel Index. Maryland State Medical Journal 1965;14: 61-5.

\section{Norton-Craft 2012}

Nordon-Craft A, Moss M, Quan D, Schenkman M. Intensive care unit-acquired weakness: implications for physical therapist management. Physical Therapy 2012;92 (12):1494-506.

Ohtake 2012

Ohtake PJ, Strasser DC, Needham DM. Rehabilitation for people with critical illness: taking the next steps. Physical Therapy 2012;92(12):1484-8.

RevMan 2012

The Nordic Cochrane Centre, The Cochrane Collaboration. Review Manager (RevMan). 5.2. Copenhagen: The Nordic Cochrane Centre, The Cochrane Collaboration, 2012.

\section{Roach 1988}

Roach KE, Van Dillen LR. Development of an Acute Care Index of Functional status for patients with neurologic impairment. Physical Therapy 1988;68(7):1102-8.

\section{Skinner 2009}

Skinner EH, Berney S, Warrillow S, Denehy L.

Development of a physical function outcome measure (PFIT) and a pilot exercise training protocol for use in intensive care. Critical Care and Resuscitation 2009;11(2): $110-5$.

\section{Skinner 2011}

Skinner EH, Warrillow S, Denehy L. Health-related quality of life in Australian survivors of critical illness. Critical Care Medicine 2011;39(8):1896-905.

\section{Stevens 2007}

Stevens RD, Dowdy DW, Michaels RK, Mendez-Tellez PA, Pronovost PJ, Needham DM. Neuromuscular dysfunction acquired in critical illness: a systematic review. Intensive Care Medicine 2007;33(11):1876-91.

\section{Zanni 2010}

Zanni JM, Korupolu R, Fan E, Pradhan P, Janjua K, Palmer $\mathrm{JB}$, et al.Rehabilitation therapy and outcomes in acute respiratory failure: an observational pilot project. Journal of Critical Care 2010;25(2):254-62.

* Indicates the major publication for the study

\section{A P P E N D I C E S}

\section{Appendix I. MEDLINE (OvidSP) search strategy}

Database: Ovid MEDLINE(R) <1946 to June Week 4 2013>

Search Strategy:

1 randomized controlled trial.pt. (378039)

2 controlled clinical trial.pt. (88553)

3 randomized.ab. (275457)

4 placebo.ab. (149266)

5 drug therapy.fs. (1722456)

6 randomly.ab. (193185)

7 trial.ab. (287286)

8 groups.ab. (1239381)

9 or/1-8 (3208243)

10 exp animals/ not humans.sh. (3996981)

119 not 10 (2730505)

12 Polyneuropathies/ (4193)

13 Muscle Weakness/ (5472)

14 Rhabdomyolysis/ (4264)

15 Quadriplegia/ (6952)

16 Paresis/ (4918)

17 neuromuscular manifestation\$1.tw. (88)

18 (polyneuropath\$ or myopath\$).tw. (28621)

19 polyneuromyopath\$.tw. (38)

20 neuromuscular disorder\$.tw. (3081)

Physical rehabilitation for critical illness myopathy and neuropathy (Protocol)

Copyright @ 2014 The Cochrane Collaboration. Published by John Wiley \& Sons, Ltd. 
21 neuromuscular disease\$.tw. (3989)

22 paresis.tw. (7490)

23 quadriplegia.tw. (2112)

24 weakness.tw. (33892)

25 neuromyopath\$.tw. (344)

26 motor syndrome.tw. (172)

27 muscle function.tw. (6982)

28 muscle strength/ (8259)

29 (muscle strength or respiratory failure).tw. (29931)

30 or/12-29 (127024)

31 Intensive Care/ (14924)

32 Critical Illness/ (16281)

33 intensive care.tw. (81047)

34 critical\$ ill\$.tw. (27308)

35 ventilator weaning/ (2849)

36 respiration, artificial/ (38024)

37 or/31-36 (138468)

38 electric stimulation therapy/ or electrostimulation.mp. or (electric $\$$ adj2 stimulation).tw. (59228)

39 ((leg or lower) adj2 (limb\$ or extremit\$)).tw. (60715)

4038 and 39 (873)

41 exp Exercise/ (109120)

42 physical therapy modalities/ or physiotherap\$.tw. or physical therap\$.tw. (42309)

43 exp Exercise Therapy/ (29085)

44 Musculoskeletal Manipulations/ (893)

45 Occupational Therapy/ (9889)

46 recovery of function/ (30425)

47 "Activities of Daily Living"/ (49818)

48 strength training.mp. (2517)

49 muscle training.mp. (1264)

50 (physical adj5 rehabilitation).tw. (4999)

51 or/40-50 (251838)

5211 and 30 and 37 and 51 (85)

\section{CONTRIBUTIONS OFAUTHORS}

All authors contributed to the conception and design of the protocol and approved the protocol. All authors will be involved in all stages of the review. JM will screen titles and abstracts of publications identified by the searches. MP and JM will extract trial and outcome data from the selected trials and analyse outcome data. JK and MP will assess the risk of bias in included studies. All authors will interpret the results.

\section{DECLARATIONSOF INTEREST}

None known 


\section{SOURCES OF SUPPORT}

\section{Internal sources}

- Wissenschaftliches Institut, Private Europäische Medizinische Akademie der Klinik Bavaria in Kreischa GmbH, An derWolfsschlucht 1-2, 01731 Kreischa, Germany.

- Lehrstuhl Therapiewissenschaften, SRH Fachhochschule für Gesundheit Gera gGmbH, Hermann-Drechsler-Str. 2, 07548 Gera, Germany.

- Gesundheitswissenschaften/Public Health, Medizinische Fakultät Carl Gustav Carus der TU Dresden, Fetscherstr. 74, 01307 Dresden, Germany.

- University of Southampton, UK.

\section{External sources}

- No sources of support supplied 